\title{
A LTE Uplink Scheduling Algorithm Based on Water Filling Principle
}

\author{
Zhou Yuan \\ College of Information Engineering \\ Huanghuai University \\ Zhumadian,China \\ iezy@sina.com
}

\begin{abstract}
Through the analysis of uplink scheduling process in LTE system, the common PF scheduling algorithm was improved and a new uplink schedule algorithm was proposed based on water filling principle. The new algorithm was demonstrated through the simulation for GBR traffic on OPNET platform. The result proved that the new algorithm can improve the uplink throughput of LTE system, and the user fairness can also be improved.
\end{abstract}

Keywords- LTE; Uplink scheduling; PF algorithm; OPNET ; Water filling

\section{INTRODUCTION}

Mobile communication system has developed to the 4th generation $(4 \mathrm{G})$. With the increase in the number of users and the rapid growth of large flow in mobile data services, the uplink traffic of the $4 \mathrm{G}$ mobile communication system is growing rapidly. So, how to use the limited wireless resources to meet the growing demand has become a problem to pay more attention for the mobile communication system manufacturers and operators ${ }^{[1]}$.In 4G LTE communication network, the core of LTE resource management system is resource scheduling, which is based on the uplink scheduling algorithm for different users according to the current channel quality, current traffic, resource usage and other factors, as far as possible to ensure the allocation of resources is not wasted ${ }^{22]}$. Therefore, it is an important method to improve the network performance and the throughput of the system by studying and designing the LTE uplink resource scheduling algorithm. At present, some research ${ }^{3-5]}$ has proposed the optimized scheduling algorithm of the LTE uplink resource scheduling in the academic circles. But these algorithms mostly focus on the priority of the scheduling algorithm, which is often not well balanced to the system's throughput performance and user fairness index. In this paper, proposed a LTE uplink scheduling algorithm with principle of water injection through studying the important business types of GBR system based on the existing typical uplink scheduling algorithms. The proposed algorithm not only takes into account the user fairness index, but also can better take into account the user service level and the system uplink.

\section{UPLINK SCHEDULING OF LTE AND PROCESS OF HARQ}

Fig.1 shows the flowchart of the uplink scheduling process in LTE. When there were data to send, UE issues scheduling request were transmitting to the eNB on the PRACH or SR-PUCCH; After receiving the request, eNB sended BO (Buffer Occupation) to inform UE by UL grant (uplink scheduling grant); When UE received the message, it transmitted buffer status report to the eNB to inform how much data needed to be sent; According the state report, eNB performed scheduling to UE and LCG, so it can allocate resources of the uplink channel rationally and inform UE through UL grant; Finally, UE transmitted data on their own logical channel according certain priority principle.

Fig.2 shows the timing sequence of HARQ process. The operation of uplink synchronization HARQ (hybrid automatic repeat request) can be done by the transmission of signaling ACK, NACK and the retransmission of NDI, uplink data. No more signaling support is needed at each retransmission, which only indicates that the transport of new data or the previous data retransmission through the NDI.

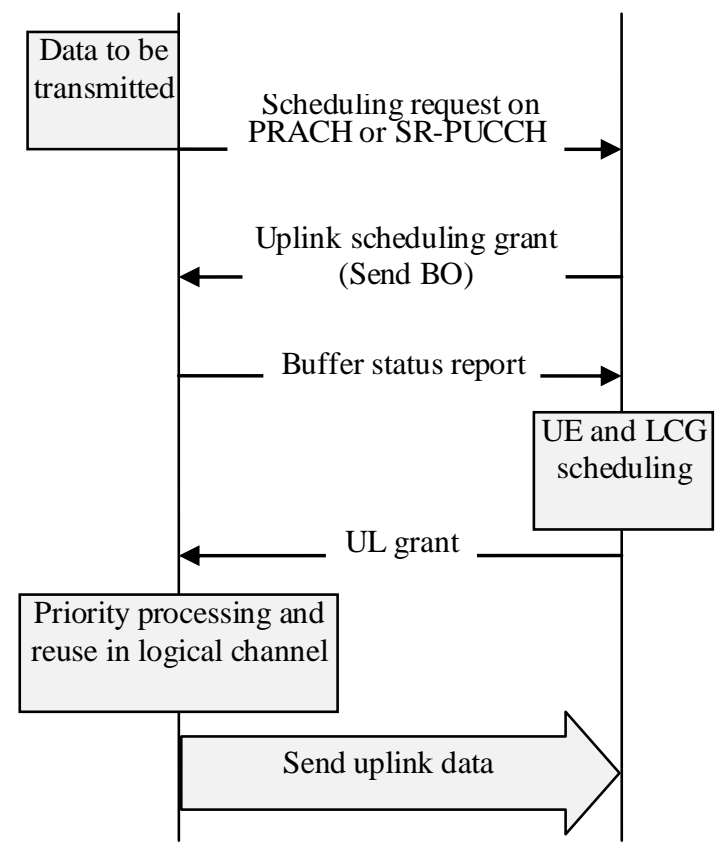

Figure 1. Flowchart of uplink scheduling 


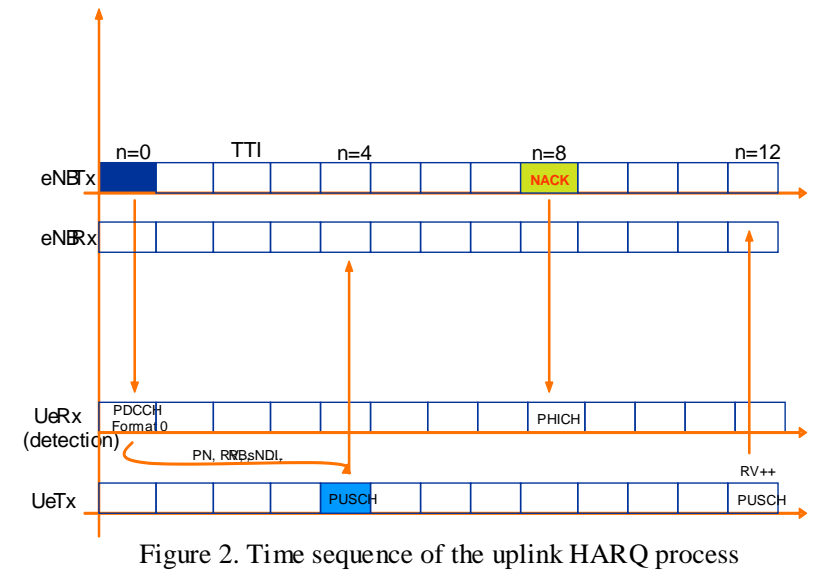

III. ALGORITHM DESIGN OF LTE UPLINK SCHEDULING

\section{A. Analysis of typical uplink scheduling algorithm}

There are three typical packet scheduling algorithms in LTE system, which are Max C/I algorithm, RR (Round Robin, RR) algorithm and PF (Proportional Fair, PF) algorithm. From the literature ${ }^{[2]}$, it can be seen that the Max C/I scheduling algorithm can reach the maximum throughput, but it is difficult to guarantee the fairness of the user; RR algorithm's fairness is the best, but the system throughput is the lowest. PF algorithm takes into account the fairness and the system throughput, so it has been widely used in practice. In this paper, proposed a new algorithm of uplink scheduling with water injection based on the basic idea of PF algorithm and verified its performance by simulation.

\section{B. Design of scheduling algorithm based on the principle of water filling}

Description of the algorithm is illustrated with a typical GBR service as an example: Suppose a cell users waiting for scheduling request are UE1, UE2,...,UEn, the corresponding GBR service rate are GBR1, GBR2, GBR3, ..., GBRn. The unit is Kbit/s. Set up a "bucket" for each to fill water and the size of the bucket is set to BucketSize $_{i}, \mathrm{i}=1,2, \ldots, \mathrm{n}$. When scheduling, uplink scheduler need to check for the remaining water (the remaining data) in Bucket. The more the remaining water, indicating that the user should promptly dispatch which can reflect the fairness and effectiveness of scheduling. BucketSize $_{\mathrm{i}}$ can be defined by formula (1).

$$
\begin{aligned}
& \text { BucketSize }_{i}=\min \left(\text { BucketSize }_{\max },\left(G B R_{i} / 1000\right) \times 1000 \times N\right) \\
& i=1,2, \ldots, n
\end{aligned}
$$

In formula (1), BucketSize max $_{\text {in }}$ is the maximum packet size for a subframe, which can be calculated by formula(the maximum number of packets when using single antenna*the maximum rank of MIMO systems* redundancy factor). GBR $/ 1000$ is due to each subframe time length is $1 \mathrm{~ms}$ in LTE system, so it is required to transform the transmission rate per second for each sub frame rate. The "amount of water" in the Bucket is updated every time, which indicates that the packet size is required for each frame and then multiply by 1000 , which is converted the unit Kbit/s into bit/s; Parameter $\mathrm{N}$ is used to control the size of Bucket, which can configure flexibly according to the actual situation of the network. Normally,
$\mathrm{N}=$ maximum retransmission number of HARQ*10, which multiplied by 10 is because there are 10 sub frames in the LTE system. The amount of water update in the Bucket can be calculated by formula (2).

$$
\mathrm{g}(\mathrm{n}+1, \mathrm{i})=\mathrm{g}(\mathrm{n}, \mathrm{i})+(\mathrm{GBR} i / 1000) \times 1000 \times 8-\mathrm{s}(\mathrm{n}, \mathrm{i})
$$

Where, the current sub frame is $n ; g(n+1, i)$ is the amount of data which can be scheduled for the I user, the $n+1 T T I$, also known as the TTI $n+1$ can be scheduled for the user's water; $g(n, i)$ is the amount of water before the current TTI scheduling; s(n, i)is user data of the current successful TTI scheduling.

\section{Description of specific scheduling process.}

The overall process of the uplink scheduling based on the principle of water filling is as follows:

Step1: Each scheduling subframe of LTE uplink scheduler sort available resources PRB of pending subframe to scheduled. And make the mark, which is easy to dispatch;

Step2: uplink scheduler sort pending queue to scheduled, eliminate user queue which the current cache without data;

Step3:According to the scheduling algorithm of from high to low priority, the uplink scheduler adjust the scheduling queue and put high priority users in the front of the queue scheduling;

Step4: Uplink scheduler works according to the priority scheduling queue. According the remaining scheduling resource, try to complete Bucket residual water of the corresponding user as far as possible for one time;

Step5: When the current scheduling TTI has been completed, according to the upstream traffic arrival rate, each user in the scheduling queue is injected with water, and the Bucket of each user in the queue is updated.

3.2.3 Design of scheduling priority. The reasonable design of scheduling priority can improve the system throughput effectively. This algorithm combines the uplink scheduling data with the current channel quality. In addition, the priority compensation factor offset $(\mathrm{k})$ is set up to distinguish different user's scheduling levels. Where, $\mathrm{k}$ represents the user level. The specific formula is shown in formula (3).

$$
p(t, i)=\theta(t, i) \delta(t, i) \frac{C Q I(t, i)}{R(t, i)}+\operatorname{offset}(k)
$$

The value of $\mathrm{p}(\mathrm{t}, \mathrm{i})$ determines the scheduling level of the user $i$ at time $t$, which the greater the value gets, the higher priority of the user is obtained. $\theta(t, i)$ represents the residual data of the user $i$ at time $t$, which the greater the value gets, the higher timeliness of the user scheduling system is obtained; $\delta(\mathrm{t}, \mathrm{i})$ represents a business QoS level parameters, which the higher the value gets, the higher business level is obtained; CQI ( $t$, i)represents the frequency spectrum efficiency of the downlink channel of the user iI at time $t$, which indicates the quality of the channel, the greater the value gets, the higher user priority is obtained; $\mathrm{R}(\mathrm{t}$, i)represents the downlink transmission rate before the user iI is dispatched. In order to make the scheduling process more smooth, the CQI $(t, i)$ and $R(t, i)$ are used for filtering. CQI $(t, i) c a n$ be calculated by formula (4).

$$
C Q I(t, i)=(1-\alpha) \times C Q I\left(t_{1}, i\right)+\alpha \times C Q I\left(t_{2}, i\right)
$$


In form (4), CQI (t1, i)represents the user channel quality that is the latest CQI parameter; CQI ( $t 2$, i)represents the CQI parameter that has been received recently; $\alpha$ re presents the smoothing factor. $\mathrm{R}(\mathrm{t}, \mathrm{i}) \mathrm{can}$ be calculated by formula (5).

$$
R(t, i)=(1-\beta) \times R(t-1, i)+\beta \times R_{1}(t, i)
$$

In form (5), $\mathrm{R}(\mathrm{t}-1, \mathrm{i})$ represents the uplink transmission rate of the user, $\mathrm{R} 1(\mathrm{t}, \mathrm{i})$ represents the downlink transmission rate which user requests for at the current scheduling time. $\beta$ represents smoothing factor.

\section{SiMULATION AND EVALUATION OF ALGORITHM PERFORMANCE}

The new scheduling algorithm is simulated and compared with the common PF scheduling algorithm. The performance of the new scheduling algorithm is analyzed from two aspects: throughput and fairness. Fair performance analysis uses the mainstream evaluation method of academic $\left[{ }^{6-8]}\right.$, as formula (6).

$$
\text { fairness }=\frac{\left(\sum_{k=1}^{N} \text { UE Re lativeThroughput }(i)\right)^{2}}{\left(\sum_{k=1}^{N}(U E \operatorname{Re} \text { lativeThroughput }(i))^{2}\right) \times N}
$$

The relative throughput of the user $i$ is represented by the data volume and the spectral efficiency of the user transmission, as formula (7).

$$
\text { UERelativeThroughput }(i)=\frac{\text { UEThroughput }(i)}{\text { SpectrumEffiency }(i)}
$$

The network is set up as a typical 19 cell structure, and each cell is composed of three $120^{\circ}$ degree qualitative antenna sectors $^{[9-10]}$, as shown in Figure 2. Network mode is the same frequency network. The user level SINR is simulated in the wrap-around environment. In the simulation, Smoothing factor $\alpha=0.1, \beta=0.1$, offset $(\mathrm{k})=0$; Business type is GBR business; Empty packet error rate BLER is set to $5 \%$. The rest of the simulation parameters are shown in table 1.

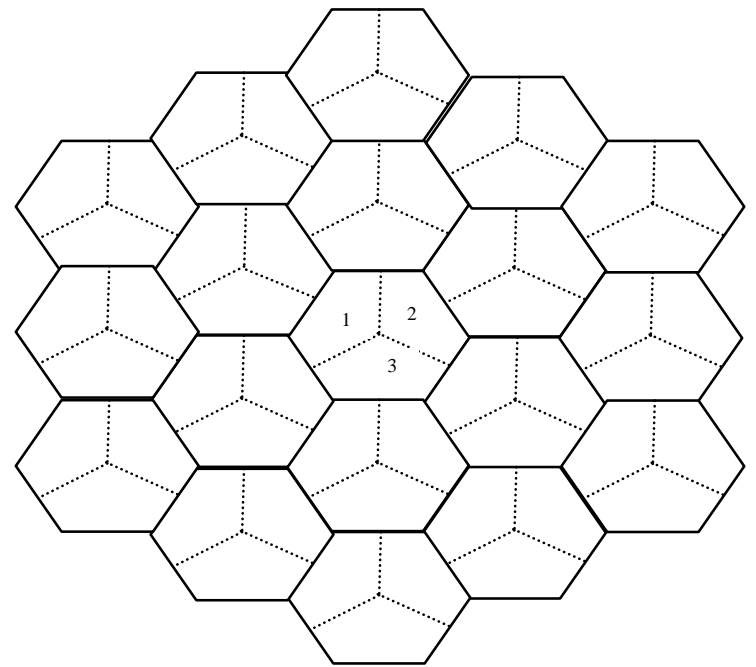

Figure 3. Topological structure diagram of simulation network
Table I. Main Parameters in Simulation

\begin{tabular}{c|c}
\hline Channel model & $\begin{array}{c}\text { According to SCM model defined in } \\
\text { 3GPP TR 25.966 }\end{array}$ \\
\hline $\begin{array}{c}\text { Antenna configuration of } \\
\text { eNodeB }\end{array}$ & $1 \mathrm{~T} 2 \mathrm{R}$ \\
\hline $\begin{array}{c}\text { Antenna configuration of } \\
\text { UE }\end{array}$ & $1 \mathrm{~T} 2 \mathrm{R}$ \\
\hline $\begin{array}{c}\text { Distance between base } \\
\text { station }\end{array}$ & $1 \mathrm{Km}$ \\
\hline Large scale fading & $128.1+37.6^{*} \log (\mathrm{d}) \mathrm{d}: \mathrm{Km}$ \\
\hline $\begin{array}{c}\text { Users location in cell } \\
\text { set value }\end{array}$ \\
\hline $\begin{array}{c}\text { System bandwidth in } \\
\text { simulation }\end{array}$ & $10 \mathrm{MHz}$ \\
\hline $\begin{array}{c}\text { LTE parameters } \\
\text { configuration }\end{array}$ & $\begin{array}{c}\text { Agreement with the definition in } \\
\text { 3GPP TS and 3GPP TR }\end{array}$ \\
\hline
\end{tabular}

The simulation platform is $O P E N T-14.0$, as 5UE, 10UE, 15UE,20UE, 25UE in one cell $\left[{ }^{11]}\right.$, using PF algorithm and the new algorithm of this paper to evaluate the impact of two scheduling algorithms on the network traffic and the fairness of users. Simulation results are shown in Figure 4 and Figure 5. From the results of the system simulation in Figure 3, the PF scheduling algorithm based on the principle of water injection is superior to the classical scheduling algorithm in throughput performance. In LTE system empty packet error rate BLER is 5\%. When the number of users is small, two algorithms of the throughput difference, the two algorithms throughput increases with the increase of the number of users and gradually reached stable network traffic, the LTE cell capacity reached equilibrium. In the figure show the approximate to a straight line. In the process of the new algorithm, the throughput of the system is always greater than that of the classical PF algorithm, which shows that the new algorithm can effectively improve the system throughput.

It can be seen fairness analysis from Figure 5, when the network load is relatively light, the user's fairness index is better in the GBR scheduling, then the PF scheduling algorithm can not achieve the corresponding performance. With the increase of the network users number, system load increases, its fairness performance of the GBR scheduling become slightly lower, but still better than PF scheduling algorithm.

BLER $=5 \%$

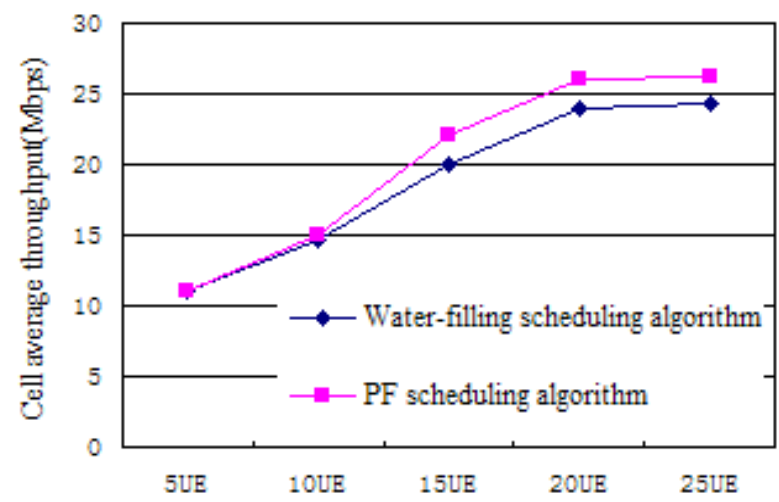

Figure 4. Throughput performance comparison of two scheduling algorithms 


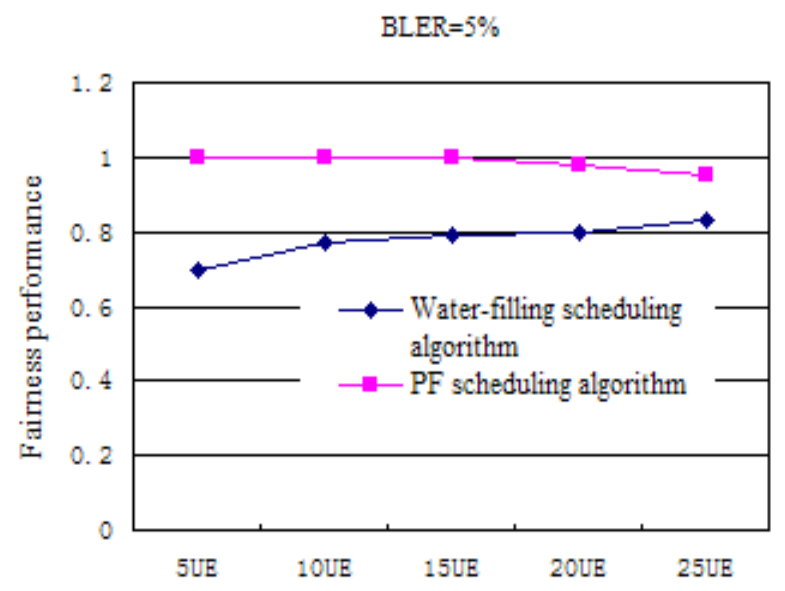

Figure 5. Fairness performance comparison of two scheduling algorithms

\section{CONCLUSONS}

In the paper, the uplink resource scheduling algorithm based on the principle of water filling is studied. The typical PF algorithm is improved in the algorithm design, the user's channel condition and the historical data has been considered, mean while the scheduling difference of different user level is realized by setting priority bias. The experimental result proved that the new algorithm can improve the uplink throughput of LTE system, and the user fairness can also be improved.

\section{ACKNOWLEDGMENT}

Key scientific research project of Henan higher education (16A510019)

Enhancement project of scientific research capacity of Huanghuai University (201512705)

\section{REFERENCES}

[1] Zou Guoqi, $\mathrm{Xu}$ Jing. Cooperative scheduling and power allocation scheme based on user clustering in LTE uplink[J]. Journal of University of Science and Technology of China, 2015,45(4):294-301

[2] Zhou Yuan, Yao Ru-xian. A LTE Uplink Scheduling Algorithm Based on Pennalty Factor[J]. Video Engineering,2014,38(11):122125

[3] Hamza A S, Khalifa S S, Hamza H S. A Survey on inter-cell interference coordination techniques in OFDMA-based cellular networks[J]. IEEE Communications Surveys \& Tutorials, 2013,15(4):1642-1670

[4] Pateromichelakis E, Shariat M, Qussus A. On the evolution of multi-cell scheduling in 3GPP LTE/LTE-A [J].IEEE Communications Surveys \& Tutorials, 2013,15(2):701-717

[5] Wang An-chun, Xiao Liang, Zhou Shi-dong,etal. Dynamic Resource Management in the fourth Generation Wireless System[C]// ICCT2003, Beijing: IEEE, 2003, vol.2,pp.1095-1098

[6] F. Capozzi, G. Piro, L. Grieco. Downlink packet scheduling in lte cellular networks: Key design issues and a survey[J].IEEE Communications Surveys \&Tutorials, 2013,15(2):678-700

[7] W. Saad, Z. Dawy, S. Sharafeddine. A utility-based algorithm forjoint uplink/downlink scheduling in wireless cellular networks[J].Journalof Network and Computer Applications, 2012, 35(1):348-356

[8] Najah Abu-Ali, Abd-Elhamid M. Taha, Mohamed Salah. Uplink Scheduling in LTE and LTE-Advanced: Tutorial, Survey and Evaluation Framewor[J]. IEEE Communications Surveys \&Tutorials, 2014,16(3):1239-1265

[9] Niu J P, Lee D, Su T. Multi-cell cooperative scheduling for uplink SC-FDMA systems[C].//24th International Symposium on Personal Indoor and Mobile Radio Communications. London,UK:IEEE Press,2013:1582-1586

[10] Jalali. Data Throughput of CDMA-HDR a High Efficiency-High Data Rate Personal Communication Wireless System.IEEE 51st, 2000.3,Tokoy,Japan,vol.3,pp.1854-1860

[11] Safa H, Tohme K. LTE uplink scheduling algorithms: performance and chanllenges[J]. Telecommunications(ICT), 2012(8):1-6. 